\title{
Myeloproliferative and lymphoproliferative malignancies occurring in the same patient: a nationwide discovery cohort
}

\author{
Johanne M. Holst, ${ }^{1,2}$ Trine L. Plesner, ${ }^{3}$ Martin B. Pedersen, ${ }^{1}$ Henrik \\ Frederiksen, ${ }^{4}$ Michael B. Møller, ${ }^{5}$ Michael R. Clausen, ${ }^{1,2}$ Marcus C. Hansen, ${ }^{1,2}$ \\ Stephen Jacques Hamilton-Dutoit, ${ }^{6}$ Peter Nørgaard, ${ }^{7}$ Preben Johansen, ${ }^{8}$ Tobias \\ Ramm Eberlein, ${ }^{9}$ Bo K. Mortensen, ${ }^{10}$ Gustav Mathiasen, ${ }^{11}$ Andreas Øvlisen, ${ }^{12}$ \\ Rui Wang, ${ }^{13}$ Chao Wang, ${ }^{14}$ Weiwei Zhang, ${ }^{15}$ Hans Beier Ommen, ${ }^{1}$ Jesper \\ Stentoft, ${ }^{1}$ Maja Ludvigsen, ${ }^{1,2}$ Wayne Tam,${ }^{13}$ Wing C. Chan, ${ }^{14}$ Giorgio Inghirami ${ }^{13}$ \\ and Francesco d'Amore ${ }^{1,2}$
}

Haematologica 2020

Volume 105(10):2432-2439

\section{Correspondence:}

FRANCESCO D'AMORE

frandamo@rm.dk

Received: May 1, 2019.

Accepted: November 26, 2019.

Pre-published: November 28, 2019.

doi:10.3324/haematol.2019.225839

(C)2020 Ferrata Storti Foundation

Material published in Haematologica is covered by copyright. All rights are reserved to the Ferrata Storti Foundation. Use of published material is allowed under the following terms and conditions:

https://creativecommons.org/licenses/by-nc/4.0/legalcode. Copies of published material are allowed for personal or internal use. Sharing published material for non-commercial purposes is subject to the following conditions:

https://creativecommons.org/licenses/by-nc/4.0/legalcode, sect. 3. Reproducing and sharing published material for commercial purposes is not allowed without permission in writing from the publisher.
${ }^{1}$ Department of Hematology, Aarhus University Hospital, Aarhus, Denmark; '2Department of Clinical Medicine, Aarhus University, Aarhus, Denmark; 'Department of Pathology, Rigshospitalet, Copenhagen, Denmark; ${ }^{4}$ Department of Hematology, Odense University Hospital, Odense, Denmark; ${ }^{5}$ Department of Pathology, Odense University Hospital, Odense, Denmark; ${ }^{\circ}$ nstitute of Pathology, Aarhus University Hospital, Aarhus, Denmark; ${ }^{7}$ Department of Pathology, Herlev Hospital, Copenhagen, Denmark; ${ }^{8}$ Department of Pathology, Aalborg University Hospital, Aalborg, Denmark; ' ${ }^{\circ}$ Department of Hematology, Regional Hospital West Jutland, Holstebro, Denmark; ${ }^{10}$ Department of Hematology, Herlev Hospital, Herlev, Denmark; ${ }^{11}$ Department of Hematology, Roskilde Hospital, Roskilde, Denmark; ${ }^{12}$ Department of Hematology, Aalborg University Hospital, Aalborg, Denmark; ${ }^{13}$ Department of Pathology and Laboratory Medicine, Weill Cornell Medicine, New York City, NY, USA; ${ }^{14}$ Department of Pathology, City of Hope Medical Center, Duarte, CA, USA and ${ }^{15}$ Department of Pathology and Microbiology, University of Nebraska Medical Center, Omaha, NE, USA

\section{ABSTRACT}

M yeloid and lymphoid malignancies are postulated to have distinct pathogenic mechanisms. The recent observation that patients with a myeloproliferative neoplasm have an increased risk of developing lymphoproliferative malignancies has challenged this assumption. We collected a nationwide cohort of patients with both malignancies. Patients diagnosed between 1990 and 2015 were identified through the national Danish Pathology Registry. We identified 599 patients with a myeloproliferative neoplasm and a concomitant or subsequent diagnosis of lymphoma. Histopathological review of the diagnostic samples from each patient led to a final cohort of 97 individuals with confirmed dual diagnoses of myeloproliferative neoplasm and lymphoma. The age range at diagnosis of these individuals was 19-94 years (median: 71 years). To avoid the inclusion of cases of therapy-induced myeloproliferative neoplasm occurring in patients previously treated for lymphoma, only patients with myeloproliferative neoplasm diagnosed unequivocally before the development of lymphoma were included. The average time interval between the diagnoses of the two malignancies was 1.5 years. In the majority of patients (90\%) both diagnoses were established within 5 years of each other. Among the lymphoma entities, the frequency of peripheral T-cell lymphomas was markedly increased. Interestingly, all but one of the T-cell lymphomas were of angioimmunoblastic type. These findings suggest that a myeloproliferative neoplasm and lymphoproliferative malignancy developing in the same patient may have common pathogenic events, possibly already at the progenitor level. We believe that the molecular characterization of the newly developed biorepository will help to highlight the mechanisms driving the genesis and clonal evolution of these hematopoietic malignancies. 


\section{Introduction}

The diagnosis of multiple clonal hematologic neoplasms in the same patient is considered to be rare. Furthermore, when it does occur, it is unclear whether the individual disorders are pathogenetically related, e.i., sharing common driver mutations, or whether they simply reflect independently developed random events.

Studies of tumor DNA from patients with angioimmunoblastic T-cell lymphoma (AITL) have identified genomic changes which can be detected in the early hematopoietic stem cell precursors. ${ }^{1-5}$ These alterations include changes in IDH2, TET2, and DNMT3A, and are commonly seen in myeloid malignancies. Acquired somatic mutation in the Janus kinase 2 (JAK2) gene plays an essential role in the development of myeloproliferative neoplasms (MPN). Notably, this mutation has also been detected in some lymphoid malignancies (LM). ${ }^{67}$ These findings have prompted the hypothesis that some genomic changes in early hematopoietic stem cell precursors may predispose to and could drive the development of both MPN and LM.

MPN are clonal hematopoietic stem cell disorders characterized by proliferation of one or more of the myeloidderived cell lineages. They include essential thrombocythemia (ET), polycythemia vera (PV), primary myelofibrosis (PMF), chronic myeloid leukemia (CML) and MPNunclassifiable (MPN-U) ${ }^{8}$ According to the database of cancer statistics for Nordic countries (NORDCAN; wWwdep.iarc.fr/NORDCAN/English/frame.asp), the age-adjusted incidence rate of CML in Denmark is $0.95 / 100,000$ and that for the other chronic myeloproliferative entities (PV, ET, PMF, MPN-U; taken as one group) 4.05/100,000. In general, MPN are slowly progressing diseases which are, however, capable of transformation to severe bone marrow failure or acute leukemia. ${ }^{8}$ Recent epidemiological studies have shown an increased risk of developing other types of malignancy in patients with MPN. ${ }^{9,10}$ In particular, the risk of also developing LM is significantly increased compared with the risk in a sex- and age-matched background population.

Dual diagnoses of MPN and LM in the same patient have previously been described in both individual case reports and in small case series. ${ }^{11}$ However, a more substantial, population-based evaluation of this phenomenon, together with a biorepository of tumor specimens from such patients, has not yet been reported. The aim of our study was to identify and characterize a nationwide cohort of Danish patients with dual diagnoses of myeloproliferative and lymphoproliferative malignancies. We describe what we believe to be the largest series of patients with diagnoses of both MPN and LM, with particular emphasis on the establishment of the cohort, the histopathological classification of the malignancies, and description of the clinical characteristics of the patients.

\section{Methods}

\section{Cohort identification}

Patients diagnosed with both MPN and LM within the period 1977-2015 were identified through the national Danish Pathology Registry (DPR). ${ }^{12}$ The DPR is a nationwide register that records data on all pathology specimens from patients in Denmark. The registry and its associated database are updated daily. Since essentially all pathology investigations in Denmark are performed within the tax-funded public health system, the coverage of the DPR is close to $100 \%$. The registry includes detailed variables related to patients, specimens and workload. All Danish citizens and residents are assigned a unique identifier, the civil personal registration number, at birth or immigration..$^{13}$ Using this registration number, a patient's data in the DPR can be linked to the many other Danish clinical databases. In addition, each specimen identified via the DPR can be linked to the available formalin-fixed, paraffin-embedded tissue biopsies stored in the diagnostic archives of the Danish pathology departments, allowing identification, location and retrieval of relevant primary diagnostic tissue specimens from the cohort patients.

The MPN diagnoses include PV, ET, PMF, CML, and MPN-U. In order to exclude secondary myelodysplasias/MPN occurring as a result of previous treatment for LM, only patients diagnosed with either both diseases concomitantly (i.e., diagnosed no more than 6 months apart) or with MPN first and LM subsequently, were selected for further histopathological revision. Because of the low number of samples available for the period 1977 to 1990 and the poor tissue quality of the older samples, only specimens from patients diagnosed in 1990 or later were included in the final cohort.

The study was approved by The Central Denmark Region Committees on Health Research Ethics (record n. 1-10-72-161-15) and the Danish Data Protection Agency (record n. 1-16-02-42015), and it was conducted in compliance with the principles of the Helsinki Declaration.

\section{Data sources}

All data sources were linked using the unique patient-specific civil personal registration number. Clinical data were obtained from the population-based Danish National Lymphoma Registry $(\mathrm{LYFO})^{14}$ and supplemented by medical records.

For comparison of overall survival, a diffuse large B-cell lymphoma (DLBCL) reference cohort matched for age, sex, and the International Prognostic Index (IPI) was identified and randomly selected from LYFO ( $n=100)$. For AITL patients, a reference cohort previously described by Pedersen et al. was used $(n=25) .{ }^{15}$

\section{Tissue collection and histological revision}

All specimens were pre-therapeutic biopsies from patients diagnosed with both MPN and LM. Formalin-fixed, paraffin-embedded tissue specimens were collected from the archives at 15 different Danish pathology departments. After careful evaluation of the original pathology reports, new hematoxylin and eosin-stained sections were cut from the study paraffin blocks and the histopathological diagnoses were reviewed. If necessary, supplementary immunohistochemical stains were assessed, in addition to those originally performed. Samples were reviewed by an experienced hematopathologist (TLP) at a tertiary referral center according to the 2017 revision of the 2008 World Health Organization Classification of Tumours of Haematopoietic and Lymphoid Tissues. ${ }^{8}$ AITL tumors were specifically tested for wellknown recurrent mutations in IDH2, TET2, DNMT3A, and RHOA genes.

\section{Statistical methods}

Outcomes were described by overall survival, defined as the time interval from LM diagnosis to last follow-up or death from any cause. Overall survival estimates were calculated according to the Kaplan-Meier method and compared using the log-rank test. All statistical analyses were performed using STATA version IC 14.1 (StataCorp, College Station, TX, USA). 


\section{Results}

\section{Establishment of the cohort}

The algorithm leading to the establishment of the cohort is shown in Figure 1. In total, 26,736 patients with MPN were identified. Of these, 1,524 had an additional registered diagnosis of concomitant or subsequent LM. If multiple biopsies from the MPN and/or LM diagnosis of a given patient were taken at different times the patient would end up with multiple registrations in the DPR. These redundant registrations were omitted $(n=925)$. Patients with diagnoses of MPN and/or LM, which were unconfirmed after histopathological review ( $\mathrm{n}=344)$, were excluded. A group of 67 patients diagnosed with LM prior to the MPN were also excluded, as were 18 patients diagnosed before 1990. Thus, diagnostic tissue specimens from 170 patients were retrieved for histopathological validation. Of these, 97 patients were confirmed to have both MPN and LM and represent the final cohort of the study.

\section{Demographic and clinico-pathological features}

Table 1 summarizes the demographic characteristics of the patients. No major differences in sex distribution were observed, either overall or at subtype-specific level. The age at MPN diagnosis was for most patients within the sixth and seventh decades of life with the exception of patients with lymphoblastic lymphoma (LBL), who were significantly younger (median 33 years; interquartile range, 22-57 years). The overall average time between the diagnoses of MPN and LM was 1.5 years and in the majority of the patients $(90 \%)$ both diagnoses were established within 5 years of each other.

Table 2 shows a cross tabulation between MPN and LM diagnoses. The most frequent LM diagnoses were chronic lymphocytic leukemia (CLL, 32\%) and DLBCL (21\%), in line with these being two of the more common LM entities in the general population.

Peripheral T-cell lymphomas (PTCL) usually account for approximately $10 \%$ of all lymphomas, which is in line with the relative frequencies seen in our study.

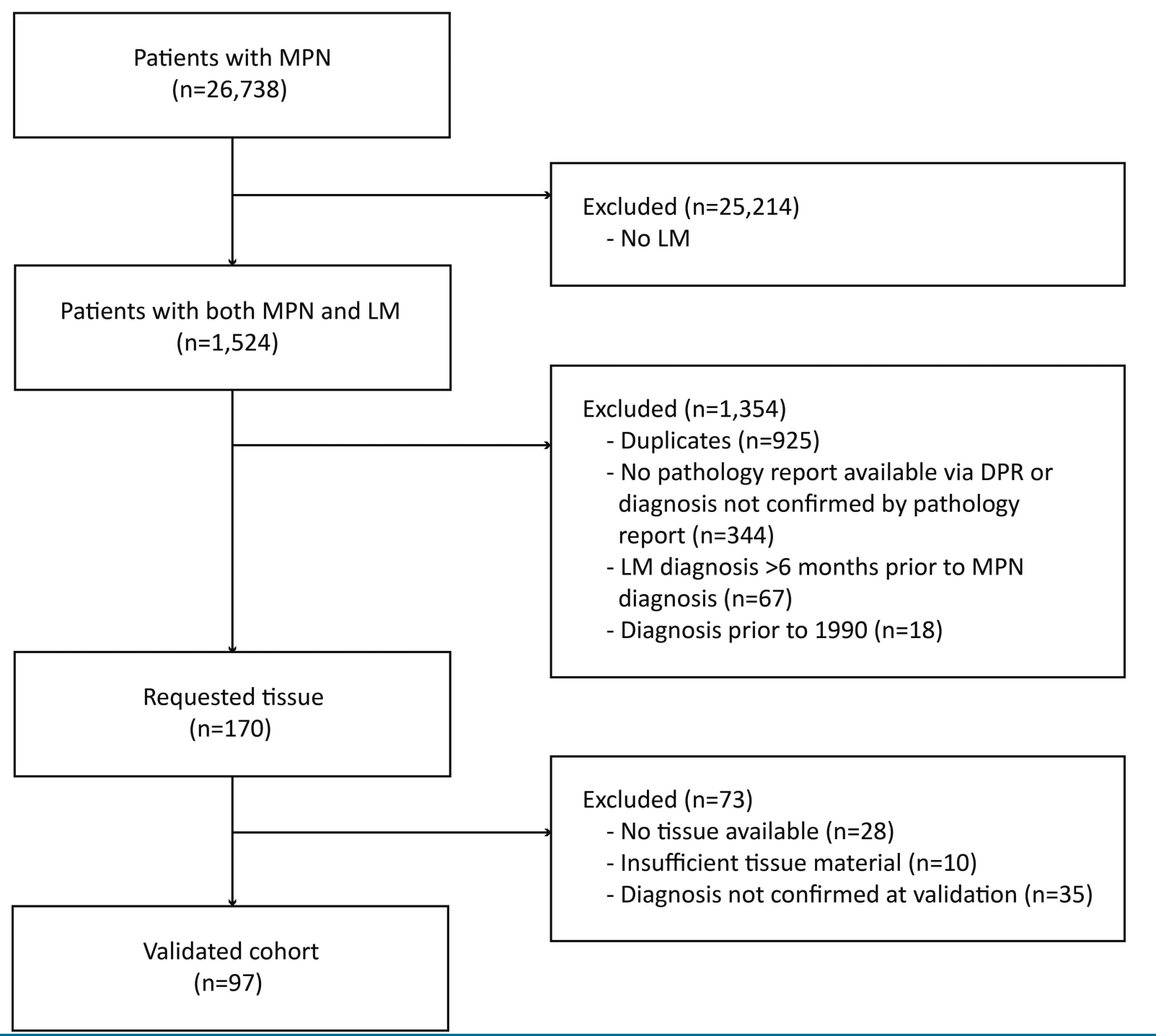

Figure 1. Flowchart illustrating the establishment of the cohort. DPR: the Danish pathology register; MPN: myeloproliferative neoplasms; LM: lymphoproliferative malignancy. 
Interestingly, AITL patients accounted for $89 \%$ (8 out of 9) of the MPN-associated PTCL, an unexpectedly high frequency (5- to 7-fold) for this specific entity. AITL tumors frequently harbored mutations of TET2 (60\%), IDH2 $(60 \%), D N M T 3 A(60 \%)$, and $R H O A(80 \%)$ genes. All five patients with LBL (B-cell lineage, $n=2$; T-cell lineage, $n=2$; and unclassifiable, $\mathrm{n}=1$ ) were associated with a pre-existing CML, probably being cases of lymphoid blast crises on a CML background.

Overall, 64\% ( $n=62)$ of the MPN patients were diagnosed with a concurrent LM, while the remaining $36 \%$ $(n=35)$ developed LM subsequently. Figure 2 illustrates the chronological occurrence of MPN and LM in each patient with PTCL, DLBCL, and CLL. The majority ( $n=23 ; 77 \%)$ of CLL patients were diagnosed with both malignancies concurrently. In contrast, most DLBCL ( $\mathrm{n}=11 ; 55 \%)$ and, even more strikingly, most PTCL patients ( $n=6 ; 67 \%$ ) were diagnosed at a mean time interval of between 1 to 3 years after the MPN diagnosis (1.5 years for DLBCL and 2.8 years for PTCL).

\section{Outcome in selected lymphoma subtypes}

In a survival analysis, patients with both MPN and DLBCL had an inferior outcome compared with an age-, sex-, and IPI-matched DLBCL reference cohort [hazard ratio $(\mathrm{HR})=1.9,95 \%$ confidence interval $(95 \% \mathrm{CI}): 1.1-3.3$;
$P<0.02]$ (Figure 3A). The 5-year overall survival rate of the patients with MPN+DLBCL was 19\% (95\% CI: 5-39\%) as compared with 34\% (95\% CI: $24-43 \%$ ) for the DLBCL patients of the matched reference cohort.

In contrast, no difference was found in outcomes between patients with both MPN and PTCL and patients with 'AITL only' (Figure 3B).

The 5-year overall survival of the patients with MPN+CLL was 65\% (95\% CI: 45-79\%). Historical data from the Danish CLL group showed a corresponding value for 'CLL only' patients diagnosed in the period 20122017 of $79 \%$ (95\% CI: $77-81 \%) .{ }^{16}$

\section{Discussion}

In establishing this population-based cohort, we identified a substantial number of patients diagnosed with myeloid malignancies, who were also diagnosed, either at the same time or later, with a lymphoid neoplasia. Among these lymphoid neoplasias, AITL were found at a much higher frequency than expected. We believe that this represents the largest reported cohort of patients with dual MPN and LM diagnoses, and that the associated biorepository is unique for its potential to foster the recognition of driver pathogenetic aberrations as well as the hierarchical

Table 1. Demographic features of the study population.

\begin{tabular}{|c|c|c|c|c|c|c|}
\hline & All & CLL & DLBCL & PTCL & WM & LBL \\
\hline Number & 97 & 31 & 20 & 9 & 10 & 5 \\
\hline Male/female ratio & 1.1 & 0.9 & 1.0 & 0.8 & 2.3 & 4.0 \\
\hline $\begin{array}{l}\text { Age, years } \\
\text { At MPN diagnosis, range } \\
\text { At MPN diagnosis, median } \\
\text { At MPN diagnosis, IQR }\end{array}$ & $\begin{array}{c}19-94 \\
71 \\
63-79\end{array}$ & $\begin{array}{c}57-88 \\
72 \\
63-81\end{array}$ & $\begin{array}{c}52-88 \\
71 \\
66-79\end{array}$ & $\begin{array}{c}60-82 \\
67 \\
64-73\end{array}$ & $\begin{array}{c}59-81 \\
71 \\
64-77\end{array}$ & $\begin{array}{c}19-57 \\
33 \\
22-57\end{array}$ \\
\hline $\begin{array}{l}\text { Time between the MPN and } \\
\text { LM diagnoses (mean), years }\end{array}$ & 1.5 & 1.4 & 2.4 & 2.8 & 1.4 & 1.7 \\
\hline
\end{tabular}

CLL: chronic lymphocytic leukemia/small lymphocytic lymphoma; DLBCL: diffuse large B-cell lymphoma; PTCL: peripheral T-cell lymphoma;WM:Waldenström macroglobulinemia; MPN: myeloproliferative neoplasms; IQR: interquartile range; LBL: lymphoblastic lymphoma.

Table 2. Overview of the associated myeloproliferative and lymphoproliferative malignancies.

\begin{tabular}{|c|c|c|c|c|c|c|}
\hline & PV & ET & PMF & CML & MPN-U & Total \\
\hline Chronic lymphocytic leukemia & 8 & 6 & 2 & 1 & 14 & 31 \\
\hline Diffuse large B-cell lymphoma & 8 & 2 & 2 & 2 & 6 & 20 \\
\hline Low grade B-cell lymphoma - NOS & 4 & - & 3 & - & 4 & 11 \\
\hline $\begin{array}{l}\text { Peripheral T-cell lymphoma } \\
\text { Anaplastic large cell lymphoma } \\
\text { Angioimmunoblastic T-cell lymphoma }\end{array}$ & $\begin{array}{l}- \\
2\end{array}$ & $\begin{array}{l}- \\
2\end{array}$ & $\begin{array}{l}1 \\
1\end{array}$ & - & $\begin{array}{l}- \\
3\end{array}$ & $\begin{array}{l}1 \\
8\end{array}$ \\
\hline Waldenström macroglobulinemia & - & 1 & 4 & 2 & 3 & 10 \\
\hline Lymphoblastic lymphoma & - & - & - & 5 & - & 5 \\
\hline Marginal zone lymphoma & - & - & 1 & - & 4 & 5 \\
\hline Hodgkin lymphoma & - & 1 & - & 1 & - & 2 \\
\hline Follicular lymphoma & - & 1 & - & 1 & - & 2 \\
\hline Mantle cell lymphoma & - & - & - & - & 1 & 1 \\
\hline Primary CNS lymphoma & - & - & - & 1 & - & 1 \\
\hline Total & 22 & 13 & 14 & 13 & 35 & 97 \\
\hline
\end{tabular}

PV: polycythemia vera; ET: essential thrombocythemia; PMF: primary myelofibrosis; CML: chronic myeloid leukemia; MPN-U, myeloproliferative neoplasms - unclassifiable; NOS: not otherwise specified; CNS: central nervous system.. 
relationship within the clonal evolution of hematologic malignancies.

Epidemiological studies have revealed that patients with MPN have an increased standardized risk of developing LM. ${ }^{9,17-20}$ Recently, a Swedish population-based study confirmed an increased risk of second malignancies in MPN patients with a hazard ratio of 2.6 (2.0-3.3) for developing lymphoma. ${ }^{10}$ However, none of the published studies indicated the frequency of different LM subtypes. Our data revealed a diverse range of different lymphoma entities. In the general population, AITL is, along with PTCL-NOS, the most common PTCL, accounting for $20-35 \%$ of all cases of PTCL in Caucasian populations. ${ }^{21,22}$ Notably, in our cohort, eight of nine PTCL cases were of angioimmunoblastic type, i.e., approximately 4-fold the expected number.
AITL has a complex clinical picture and is often diagnosed at advanced stage. ${ }^{23}$ Histopathological examination of AITL tissue usually shows a microenvironment of nonmalignant bystander cells, together with a minor population of neoplastic follicular helper T cells (TFH), which are believed to be the cell of origin of AITL. ${ }^{24}$ Recently, molecular alterations unique to AITL and to PTCL of TFH-origin have been described., ${ }^{4,25}$ Among the most frequent genomic alterations are recurrent mutations of RHOA $5,{ }^{26}$ and epigenetic modifier genes such as DNMT3A, TET2, and IDH2, 1,3,27,28 The latter are wellknown genetic lesions and were originally identified in myeloid malignancies including myelodysplastic syndromes and MPN. DNMT3A and TET2 mutations have been predominantly found in progenitors prior to T-cell and B-cell commitment, whereas RHOA and IDH2 muta-
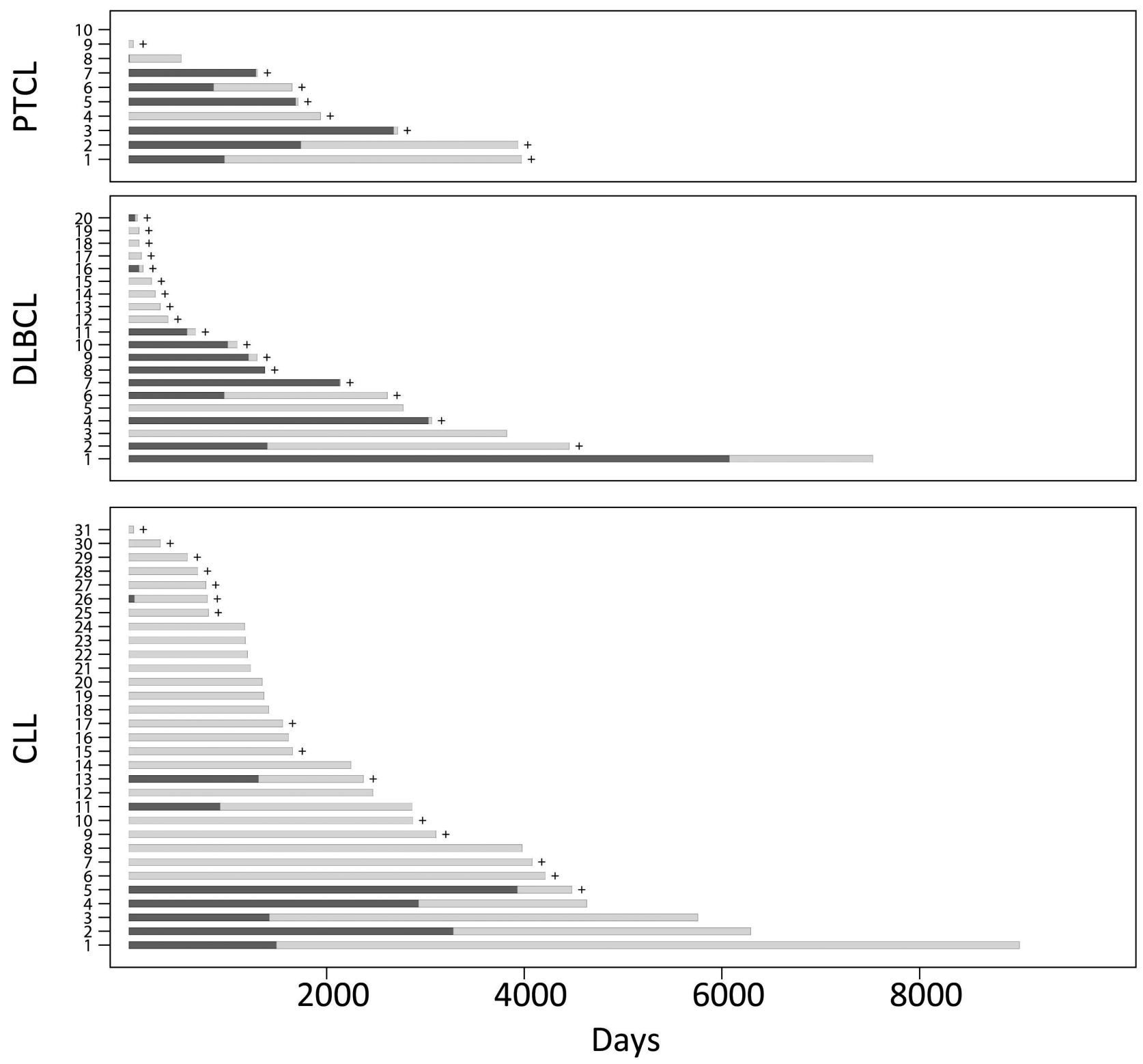

Figure 2. Swimmer plots showing, for each patient, for three selected lymphoid diagnoses the chronological occurrence of the myeloproliferative and lymphoproliferative malignancies. Each bar represents one patient in the study. Dark gray parts of the bars represent the time between the diagnosis of the myeloproliferative neoplasm and the lymphoma diagnosis. Light gray parts of the bars represent time with both diagnoses to death or last follow-up. PTCL: peripheral T-cell lymphoma; DLBCL: diffuse large B-cell lymphoma; CLL: chronic lymphocytic leukemia/small lymphocytic lymphoma. 
tions are usually found more downstream in cells that have undergone lineage specification. ${ }^{29}$ TET2 and IDH2 mutations are mutually exclusive in myeloid malignancies, but often co-exist in AITL., ${ }^{5,25}$ Interestingly, the combination of TET2 deletion together with RHOA mutation has been shown to lead to the development of AITL in mice..$^{30-32}$ In spite of an increasing number of anecdotal reports of MPN associated with the development of a variety of LM, no definitive relationship between the conditions has been established. ${ }^{33}$ However, the relatively high frequency of mutations in epigenetic modifier genes, found in both myeloid and lymphoid malignancies, could suggest a possible pathogenetic relevance of these mutations for the dual malignant transformation. Moreover, they may represent evidence of shared pathogenetic mechanisms related to hierarchical mutation steps occurring as early events in the hematopoietic neoplastic process.

Development of second malignancies in MPN patients may influence survival. ${ }^{34}$ Our study indicates that patients with both MPN and DLBCL have a worse prognosis compared with a reference cohort of patients with DLBCL alone matched for age, sex, and IPI score. This observation cannot be readily explained, but may be due to added morbidity as a consequence of multiple treatment courses related to both the myeloid and lymphoid malignancies and/or added deleterious genomic alterations at both stem cell and committed lineage-specific cell level.

A

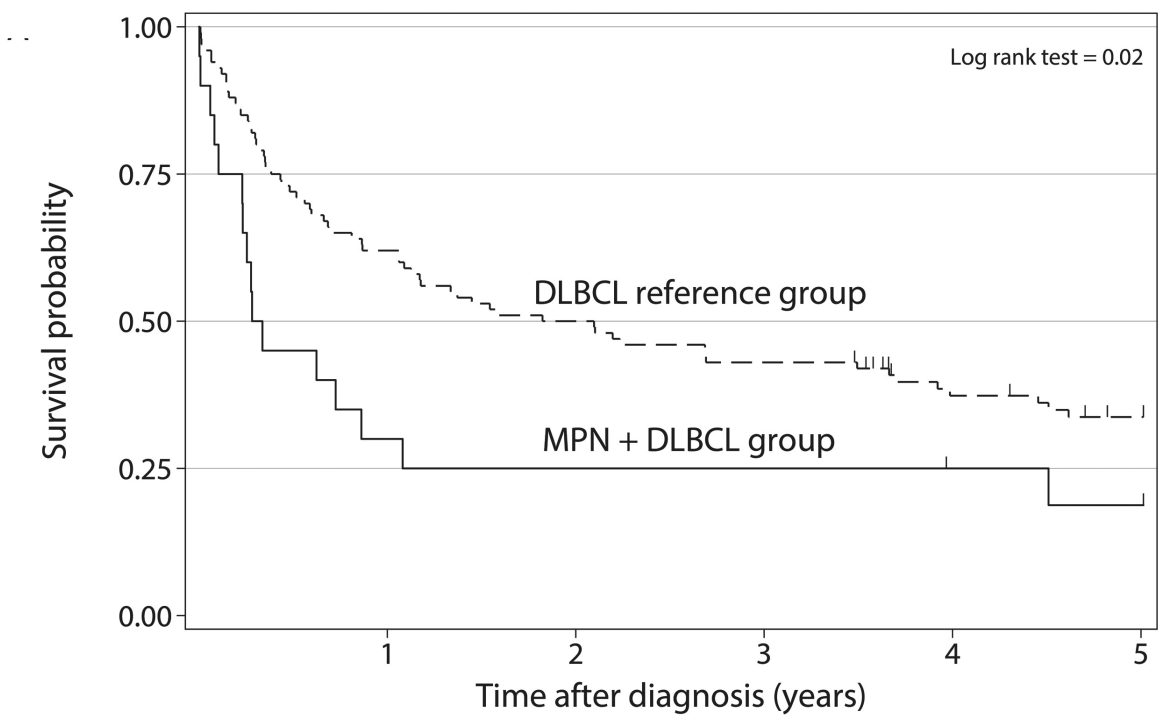

\begin{tabular}{|c|c|c|c|c|c|c|}
\hline Number at risk & & & & & & \\
\hline $\mathrm{MPN}+\mathrm{DLBCL}$ & 20 & 6 & 5 & 5 & 4 & 3 \\
\hline DLBCL ref & 100 & 62 & 50 & 43 & 32 & 26 \\
\hline
\end{tabular}

B

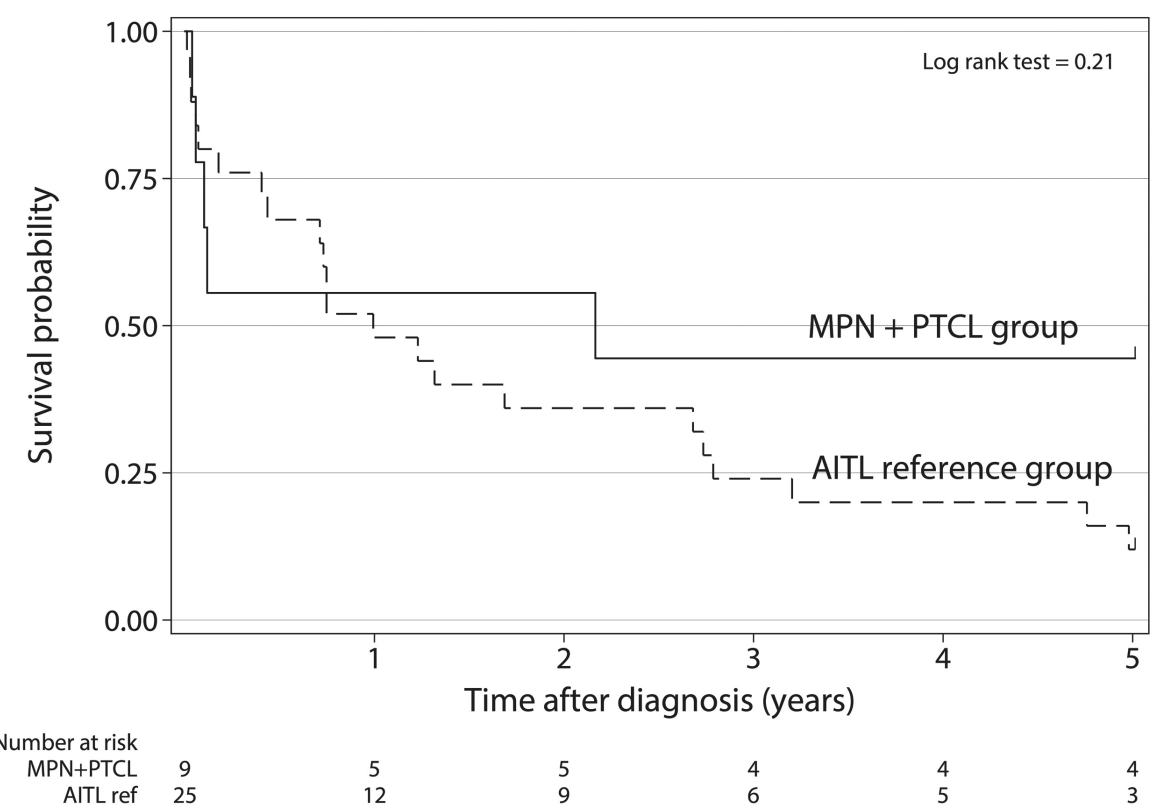

Figure 3. Survival analyses. Kaplan Meier estimates of overall survival in (A) patients with diffuse large B-cell lymphoma with and without a previous diagnosis of a myeloproliferative neoplasm and (B) patients with peripheral T-cell lymphoma with and without a previous diagnosis of a myeloproliferative neoplasm. DLBCL: diffuse large B-cell lymphoma; MPN myeloproliferative neoplasm; PTCL: peripheral T-cell lymphoma; AITL: angioimmunoblastic T-cell lymphoma. 
Conversely, no significant outcome difference was seen between patients with both MPN and AITL and a reference cohort diagnosed with AITL alone. AITL is a rare disease with a poor prognosis and with frequent relapses. Hence, our outcome analysis was expectedly hampered by the limited size of the compared cohorts, and additional studies are needed to verify this observation.

Recently, an overrepresentation of B-cell lymphomas in patients with PMF treated with JAK1/2 inhibitors has been observed. ${ }^{35}$ None of the patients with myelofibrosis in our cohort had received JAK inhibition therapy.

Observational studies based on archival material have inherent limitations regarding the completeness of available clinical information and the validity of the histopathological diagnoses. Our study is a nationwide collaboration between departments of pathology and hematology in Denmark, one major aim being to provide specimens and clinico-pathological data from a population-based cohort of patients diagnosed with both a myeloid and a lymphoid malignancy. Furthermore, all cases underwent histopathological validation including a diagnostic update according to the most recent revision of the World Health Organization classification. ${ }^{17}$ A biorepository of tissue specimens has been established and DNA extracted for genomic analysis from both the MPN and LM samples.
In conclusion, we describe here the frequency, demographics and epidemiology of the largest reported cohort of patients diagnosed with both MPN and LM. Patients with both MPN and DLBCL have poorer outcomes than those with DLBCL only. AITL is the lymphoma entity with by far the highest relative frequency of associated MPN. We hypothesize that shared genomic abnormalities may predispose to the combined or sequential development of MPN and LM in the same host. To further investigate this hypothesis, we have established a biorepository of all available specimens from this nationwide cohort. This unique material will be an invaluable resource for the performance of genomic studies to investigate the pathogenetic relationship between MPN and LM with possible novel therapeutic implications.

\section{Acknowledgments}

The authors would like to thank the collaborating pathology and hematology departments for retrieval of tissue samples and collection of clinical data. Special thanks for technical assistance to Kristina Lystlund Lauridsen, Laboratory of Molecular Pathology at Aarhus University Hospital and Joelle Racchumi, Department of Pathology and Laboratory Medicine at Weill Cornell Medicine. This work was supported by grants from Aarhus University, iLymph, SEB pension, the Danish Lymphoma Group, and the Olufgaard P. N. Kristensen Foundation.

\section{References}

1. Dobay M, Lemonnier F, Missiaglia E, et al. Integrative clinicopathological and molecular analyses of angioimmunoblastic T-cell lymphoma and other nodal lymphomas of follicular helper T-cell origin. Haematologica. 2017;201(4):e148-e151.

2. Cairns RA, Iqbal J, Lemonnier F, et al. IDH2 mutations are frequent in angioimmunoblastic T-cell lymphoma. Blood. 2012;119(8):1901-1903.

3. Lemonnier F, Couronne L, Parrens M, et al. Recurrent TET2 mutations in peripheral Tcell lymphomas correlate with TFH-like features and adverse clinical parameters. Blood. 2012;120(7):1466-1469.

4. Couronne L, Bastard C, Bernard OA. TET2 and DNMT3A mutations in human T-cell lymphoma. N Engl J Med. 2012;366(1):9596.

5. Sakata-Yanagimoto M, Enami T, Yoshida $\mathrm{K}$, et al. Somatic RHOA mutation in angioimmunoblastic $\mathrm{T}$ cell lymphoma. Nat Genet. 2014;46(2):171-175.

6. Kodali S, Chen C, Rathnasabapathy C, Wang JC. JAK2 mutation in a patient with CLL with coexistent myeloproliferative neoplasm (MPN). Leuk Res. 2009;33(12): 236-239.

7. Roncero AM, López-Nieva P, CobosFernández MA, et al. Contribution of JAK2 mutations to T-cell lymphoblastic lymphoma development. Leukemia. 2016;30 (1):94-103.

8. Swerdlow S, Campo E, Harris N, et al. WHO Classification of Tumours of Haematopoietic and Lymphoid Tissues (revised 4th edition). Lyon. 2017

9. Frederiksen H, Farkas DK, Christiansen CF, Hasselbalch HC, Sørensen HT. Chronic myeloproliferative neoplasms and subsequent cancer risk: a Danish populationbased cohort study. Blood. 2011;118(25):
6515-6520

10. Landtblom AR, Bower $\mathrm{H}$, Andersson TM, et al. Second malignancies in patients with myeloproliferative neoplasms: a population-based cohort study of 9379 patients. Leukemia. 2018;32(10):2203-2210.

11. Marchetti M, Carobbio A, Capitoni E, Barbui T. Lymphoproliferative disorders in patients with chronic myeloproliferative neoplasms: a systematic review. Am J Hematol. 2018;93(5):698-703.

12. Erichsen R, Lash TL, Hamilton-Dutoit SJ, Bjerregaard B, Vyberg M, Pedersen L. Existing data sources for clinical epidemiol ogy: the Danish national pathology registry and data bank. Clin Epidemiol. 2010;2:5156.

13. Schmidt M, Pedersen L, Sørensen HT. The Danish civil registration system as a tool in epidemiology. Eur J Epidemiol. 2014;29(8): $541-549$

14. Arboe B, Josefsson P, Jørgensen J, et al. Danish national lymphoma registry. Clin Epidemiol. 2016;8:577-581.

15. Pedersen M, Hamilton-Dutoit S, Bendix K, et al. DUSP22 and TP63 rearrangements predict outcome of ALK-negative anaplastic large cell lymphoma: a Danish cohort study. Blood. 2017;130(4):554-557.

16. Danish Lymphoma Group - Annual Report 2017. http://www.lymphoma.dk/arsrapporter/

17. Rumi E, Passamonti F, Elena C, et al. Increased risk of lymphoid neoplasms in patients with myeloproliferative neoplasms: a study of 1,915 patients. Haematologica. 2011;96(3):454-458.

18. Vannucchi AM, Masala G, Antonioli E, et al. Increased risk of lymphoid neoplasms in patients with Philadelphia chromosomenegative myeloproliferative neoplasms. Cancer Epidemiol Biomarkers Prev. 2009;18(7):2068-2073.

19. Khanal N, Giri S, Upadhyay S, Shostrom VK, Pathak R, Bhatt VR. Risk of second pri- mary malignancies and survival of adult patients with polycythemia vera: a United States population-based retrospective study. Leuk Lymphoma. 2016;57(1):129-133.

20. Shrestha R, Giri S, Pathak R, Bhatt VR. Risk of second primary malignancies in a population-based study of adult patients with essential thrombocythemia. World J Clin Oncol. 2016;7(4):324-330.

21. Vose J, Armitage J, Weisenburger D. International peripheral $\mathrm{T}$-cell and natura killer/T-cell lymphoma study: pathology findings and clinical outcomes. J Clin Oncol. 2008;26(25):4124-4130.

22. Laurent C, Baron M, Amara N, et al. Impact of expert pathologic review of lymphoma diagnosis: study of patients from the French Lymphopath Network. J Clin Oncol. 2017;35(18):2008-2017.

23. Federico $M$, Rudiger $T$, Bellei $M$, et al. Clinicopathologic characteristics of angioimmunoblastic T-cell lymphoma: analysis of the International Peripheral $\mathrm{T}$ cell Lymphoma Project. J Clin Oncol. 2013;31(2):240-246.

24. de Leval L, Rickman DS, Thielen C et al. The gene expression profile of nodal peripheral T-cell lymphoma demonstrates a molecular link between angioimmunoblastic T-cell lymphoma (AITL) and follicular helper T (TFH ) cells. Blood. 2007;109(11): 4952-4963.

25. Odejide $O$, Weigert $O$, Lane AA, et al. A targeted mutational landscape of angioimmunoblastic T-cell lymphoma. Blood. 2014;123(9):1293-1296

26. Palomero T, Couronné L, Khiabanian H, et al. Recurrent mutations in epigenetic regulators, RHOA and FYN kinase in peripheral T cell lymphomas. Nat Genet. 2014;46(2): 166-170.

27. Vallois D, Dobay MPD, Morin RD, et al Activating mutations in genes related to TCR signaling in angioimmunoblastic and other follicular helper T-cell-derived lym- 
phomas. Blood. 2016;128(11):1490-1502.

28. Rosenquist R, Rosenwald A, Du M-O, et al. Clinical impact of recurrently mutated genes on lymphoma diagnostics: state-ofthe-art and beyond. Haematologica. 2016;101(9):1002-1009.

29. Nguyen TB, Sakata-Yanagimoto M, Asabe $\mathrm{Y}$, et al. Identification of cell-type-specific mutations in nodal T-cell lymphomas. Blood Cancer J. 2017;7(1):1-10.

30. Zang S, Li J, Yang H, et al. Mutations in 5methylcytosine oxidase TET2 and RhoA cooperatively disrupt T cell homeostasis. J
Clin Invest. 2017;127(8):2998-3012.

31. Ng SY, Brown L, Stevenson K, et al. RhoA G17V is sufficient to induce autoimmunity and promotes T-cell lymphomagenesis in mice. Blood. 2018;132(9):935-947.

32. Cortes JR, Ambesi-Impiombato A, Couronné L, et al. RHOA G17V induces T follicular helper cell specification and promotes lymphomagenesis. Cancer Cell. 2018;33(2):259-273.

33. Lemonnier F, Dupuis I, Sujobert P, et al. Treatment with 5-azacytidine induces a sustained response in patients with angioimmunoblastic T-cell lymphoma Blood. 2018;132(21):2305-2309.

34. Frederiksen H, Farkas DK, Christiansen CF et al. Survival of patients with chronic myeloproliferative neoplasms and new primary cancers: a population-based cohort study. Lancet Haematol. 2015;2(7):e289 e296.

35. Porpaczy E, Tripolt S, Hoelbl-Kovacic A, et al. Aggressive B-cell lymphomas in patients with myelofibrosis receiving JAK1/2 inhibitor therapy. Blood. 2018;132(7):694706. 